\title{
Systematic comparison of 1D and 2D hydrodynamic models for the assessment of hydropeaking alterations
}

\author{
Matthias Bürgler ${ }^{1}$, David F. Vetsch ${ }^{1}$, Robert Boes ${ }^{1}$, and Davide Vanzo ${ }^{1}$ \\ ${ }^{1}$ Eidgenossische Technische Hochschule Zurich Versuchsanstalt fur Wasserbau Hydrologie \\ und Glaziologie
}

February 5, 2022

\begin{abstract}
Numerical hydrodynamic models enable the simulation of hydraulic conditions under various scenarios and are thus suitable tools for hydropeaking related assessments. However, the choice of the necessary model complexity and the consequences of modelling choices are not trivial and only few guidelines exist. In this study we systematically evaluate numerical onedimensional (1D) and two-dimensional (2D) hydrodynamic models with varying spatial resolution regarding their suitability as input for hydropeaking-sensitive, ecologically relevant hydraulic parameters (ERHPs), and their computational efficiency. The considered ERHPs include the vertical dewatering velocity, the wetted area variation between base and peak flow and the bed shear stress as a proxy for macroinvertebrate drift. We then also quantified the habitat suitability of brown trout for different life stages. The evaluation is conducted for three channel planforms with morphological characteristics representative for regulated Alpine rivers, ranging from alternating bars to a braiding river morphology. Our results suggest, that while a highly resolved 1D model is sufficient for accurate predictions of the dewatering velocity and wetted area in the less complex alternating bar morphology, a 2D model is recommended for more complex wandering or braiding morphologies. For the prediction of habitat suitability and bed shear stress, a 1D model appears to be always insufficient, and a highly resolved 2D model is suggested. Reducing the spatial resolution of $2 \mathrm{D}$ models leads to computational efficiency similar to $1 \mathrm{D}$, while providing more accurate results. This study can serve as guideline for researchers and practitioners in the selection and setup of hydrodynamic models for hydropeaking.
\end{abstract}

\section{Hosted file}

Main_Text_Buergler_et_al_2022.pdf available at https://authorea.com/users/458861/articles/ 555317-systematic-comparison-of-1d-and-2d-hydrodynamic-models-for-the-assessment-ofhydropeaking-alterations 